\title{
Taste sensitivity of eating elicited by chemical stimulation of the rat hypothalamus'
}

DAVID A. BOOTH AND DAVID QUARTERMAIN

YALE UNIVERSITY

\begin{abstract}
Abstraet
An optimum dose of norepinephrine elicits in satiated rats a greater consumption of saccharine-treated mash but less of quinine-treated mash than in $3-\mathrm{hr}$. food deprived rats. These and other possible differences between deprivation eating and chemically elicited eating are in contrast to the eating elicited by electrical stimulation of the lateral hypothalamus.

Problem

The significance of the eating elicited by injections of adrenergic drugs through cannulae chronically implanted in the lateral hypothalamus (Grossman, 1962) depends on its relationship to eating elicited by deprivation. On the same reasoning Miller has emphasized the importance of the similarity between eating elicited by electrical stimulation and normal deprivation hunger (Miller, 1960). The relationship between norepinephrine-induced eating and eating to food deprivation has not received such close attention. This report describes an experiment designed to examine one aspect of this relationship, namely, the sensitivity of food intake to the taste of quinine and to the taste of saccharine.
\end{abstract}

\section{Method}

Ss were 14 male Holtzman rats, 350 gm in weight and approximately 150 days old at the time of the experiment. They were stereotaxically implanted with cannulae (21 or 23 gauge needles) aimed at the lateral hypothalamus according to the procedure described by Grossman (1962).

From one week after the operation all rats were screened for eating to norepinephrine. They were thoroughly satiated with wet mash in their home cages for $1 / 2 \mathrm{hr}$. before injection and removed to the test cages where a weighed amount of chow pellets was present with water ad lib. After a test period of $1 \mathrm{hr}$. the remaining food was carefully weighed. The amount of chow eaten by each rat after a variety of doses of norepinephrine bitartrate $(0.8-190 \mathrm{mM})$ was compared with that eaten after injection of isotonic saline. As in a previous study (Miller, Gottesman, \& Emery, 1964), $1 \mu 1$ of $64 \mathrm{mM}$ norepinephrine was optimum in eliciting most eating. Each rat was tested twice at this dose and twice with saline in an ABBA sequence. Six rats ate 1.8-4.0 gm (mean 2.5 gm) more chow after norepinephrine than after saline and were classed as "eaters" in the sense that they showed the phenomenon described by Grossman (1962) and Miller et al (1964), whatever anatomical and pharmacological mechanisms it may involve. Eight rats were "non-eaters," eating from 0.9 more to $1.6 \mathrm{gm}$ less chow (mean $0.0 \mathrm{gm}$ ) after norepinephrine than after saline.

Norepinephrine "eaters" were then tested for the effects of quinine and of saccharine on amounts of wet mash eaten after a moderate period of food deprivation as compared with amounts eaten under the influence of the drug. The "non-eaters" were also tested to provide some indication of the comparative effects of satiation and of moderate deprivation on intake.

All wet mashes were prepared immediately before use by mixing Purina laboratory chow with water (plain) or sodium saccharine solution $(3 \% \mathrm{w} / \mathrm{v})$ or quinine hydrochloride $(0.4 \% \mathrm{w} / \mathrm{v})$ in the proportion of $1 \mathrm{gm}$ of powder to $1 \mathrm{ml}$ of fluid. Ad lib plain mash was given $1 \mathrm{hr}$. before injection of norepinephrine. The deprivation condition was $3 \mathrm{hr}$. with any food, preceded by $1 \mathrm{hr}$. access to plain mash, and terminated by injection of saline. After injection a rat was returned to its home cage into which a weighed amount of saccharine-treated or quinine-treated mash had been placed. Water was available at all times. At $1 \mathrm{hr}$. after injection all remaining mash was carefully removed and weighed.

Tests with norepinephrine injection when satiated were made on the first and last of four days, and with saline injection after $3 \mathrm{hr}$. deprivation on the middle two days. Saccharine mash and quinine mash were alternated each day for each animal, half of each group receiving saccharine on the first day, and half quinine first.

\section{Results and Diseussion}

When the differences for each animal between amounts of saccharine and quinine treated mashes eaten after deprivation were subtracted from the differences after norepinephrine injection, the resulting treatment-condition interaction scores for "eaters" to norepinephrine differed reliably from scores for the "non-eaters" $(p<$.006); Mann-Whitney $U$ test). The results are plotted as mean amounts eaten for each group in Fig. 1. The best effects were obtained from the best eaters (the interaction scores for "eaters" and the amounts of dry chow eaten on the screening test showing a perfect rank order correlation). For both groups, the differences between treatments in the amounts eaten were in all cases reliable for both types of mash $(p<.05)$ and were in the direction hypothesized for greater taste sensitivity in norepinephrine eating compared with deprivation eating. The apparent differences between "eaters" and "non-eaters" in mean amounts eaten under $3 \mathrm{hr}$. depri- 


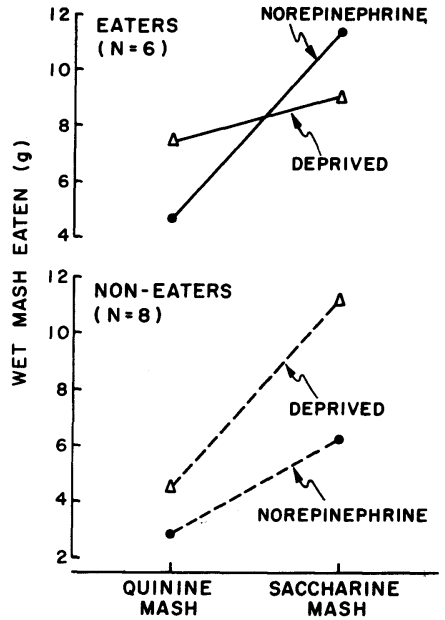

Fig. 1. Intakes of quinine- and saccharine-treated mashes during $1 \mathrm{hr}$. after $3 \mathrm{hr}$. of food deprivation or after norepinephrine injection.

vation were not statistically significant for either quinine or saccharine mash $(p<0.1)$. This of course does not exclude the possibility that "eaters" are in fact, when normally hungry, peculiarly insensitive to differences in taste, as might conceivably occur because of lesions correlated with effective cannulae, but it does make it less likely that such an effect was making a large contribution to the observed interaction.

The results strongly suggest therefore that eating to hypothalamic norepinephrine injections is qualitatively different from eating under moderate food deprivation, and more similar for example (Coons, 1965) to hyperphagia caused by some types of ventromedial hypothalamic lesion in that food intake is more sensitive to depression by quinine and elevation by saccharine than in normal hunger (Graff \& Stellar, 1962). Electrically stimulated eating, by contrast, does not appear to differ from eating under food deprivation in its sensitivity to quinine (Tenen \& Miller, 1964).

It remains to be investigated whether this effect of the drug is at all dependent on some consequence of the satiation procedure, such as stomach distension, or on the presumably limited time course of the drug's effectiveness in eliciting eating. Some further data of ours open the possibility that repeated acquaintance with the treated mashes eliminates the effect.

These results provide a quantitative basis for the impression we have gained that eating elicited by chemical stimulation of the lateral hypothalamus contrasts with that elicited by electrical stimulation or by deprivation in its marked dependence on the conditions of testing, being very unreliable, for example, when the rat is required to press a bar on a $1 \mathrm{~min}$. variable interval schedule to obtain food.

It can never be assumed that apparently normal overt behavior elicited by artificial interference with the nervous system will retain that appearance when examined under carefully varied conditions.

\section{References}

Coons, E.E. Personal communication.

Graff, H., \& Stellar, E. Hyperphagia, obesity and finickiness. J. comp. physiol. Psychol., 1962, 55, 418-424.

Grossman, S. P. Direct adrenergic and cholinergic stimulation of hypothalamic mechanisms. Amer. J. Physiol., 1962, 202, 872-882.

Miller, N. E. Motivational effects of brain stimulation and drugs. Fed. Proc., 1960, 19, 846-854.

Miller, N. E., Gottesman, K. S., \& Emery, N. Dose response to carbachol and norepinephrine in rat hypothalamus. Amer. $J$. Physiol., 1964, 206, 1384-1388.

Tenen, S. S., \& Miller, N. E. Strength of electrical stimulation of lateral hypothalamus, food deprivation and tolerance for quinine in food. J. comp. physiol. Psychol., 1964, 58, 55-62.

\section{Note}

1. This work was supported by Grant MH02949 from the National Institute of Mental Health to N. E. Miller, whom we thank for encouragement and constructive criticism. We are grateful to Mrs. A. Wechsler and Mrs. E. Love for their assistance. 